\title{
The renaissance of general relativity in Rome: Main actors, research programs and institutional structures
}

\author{
Luisa Bonolis* \\ Max Planck Institute for the History of Science, \\ Berlin, Germany \\ *E-mail: lbonolis@mpiwg-berlin.mpg.de \\ Adele La Rana \\ Sapienza University, \\ Rome, Italy \\ E-mail: Adele.LaRana@roma1.infn.it \\ Roberto Lalli \\ Max Planck Institute for the History of Science, \\ Berlin, Germany \\ E-mail: rlalli@mpiwg-berlin.mpg.de
}

\begin{abstract}
We show that the revitalization of General Relativity (GR) in Rome during the 1960s was mainly shaped by the confluence of two different research programs centered around two authoritative figures: the first was the focusing of former Levi-Civita's student Carlo Cattaneo on the mathematical problems of GR and the establishment of a research group in Rome; the second was sparked by the return in Italy of the astrophysicist Livio Gratton in 1960. We also argue that the material possibilities for a novel confluence between previously separated fields in the Roman environment was made possible by deep institutional changes and, especially, by the organizational choices and visions of Edoardo Amaldi who was leading the postwar reconstruction of Italian physics.
\end{abstract}

Keywords: General relativity; relativistic astrophysics; Tullio Levi-Civita; Giuseppe Armellini; Enrico Fermi; Carlo Cattaneo; Edoardo Amaldi; Livio Gratton.

\section{The First Phase: The interwar period}

In the late 1960s, General Relativity (GR) definitely returned to the mainstream of physics, coming into focus in connection with major astrophysical discoveries and becoming an internationally visible, highly active field of research. In order to better elucidate how this historical process - the so-called Renaissance of GR, as Clifford Will christened it — developed in Rome during the 1960s, it is necessary to put it in relation to the research and teaching of GR in the Roman institutional settings starting from the early 1920s. A summary of the interwar work in the field and some hints on the broader Italian context will allow an evaluation of the elements of continuity and discontinuity, showing how the interwar local traditions put solid bases for the re-emergence of GR in the late 1950s.

We can consider 1919 as the year in which GR officially entered Rome University. Levi-Civita, who had recently published his important work on parallel displacement, was called as Professor of Higher Analysis and then of Rational Mechanics, and did introduce some elements of the relativistic theories in his course. Contex- 
tually with Levi-Civita's appointment, a series of conferences on GR was organized at the Mathematical Seminar of Rome University, directed by Vito Volterra, with the aim to promote the theory of GR as a triumph of Ricci-Curbastro and LeviCivita's work as well as to spread the methods of tensor calculus. The conferences were inaugurated by Levi-Civita on March 8, 1919, and became the first institutional step undertaken for the diffusion of Einstein's theory in Italy - a process in large measure headed by Levi-Civita and the circle of mathematicians centered around him.

When Levi-Civita moved to Rome at the end of 1918, Enrico Fermi was beginning his university studies in Pisa. Probably influenced by the spreading of interest in relativity theories, Fermi dedicated to open problems of special relativity and GR his first four works written between 1921 and 1922, when he was still a student in Pisa. The third and most important of Fermi's contributions to relativity theories — which included the formulation of what is now known as the FermiWalker transport - was presented at the Accademia dei Lincei by the astronomer Giuseppe Armellini in January 1922. Armellini, who was Fermi's professor of Theoretical Geodesy in Pisa, was one of the few Italian astronomers who promoted and defended GR against his more conservative colleagues. In 1922, Armellini was appointed professor of Theoretical Astronomy and director of the Observatory in Rome. With Armellini and Levi-Civita, Rome became the uncontested center of GR in Italy in the interwar period.

Although his most important works concerned relativity theories, in July 1922 Fermi graduated with an experimental thesis on X ray crystallography. His friend Enrico Persico, who had got his laurea degree in physics in fall 1921, in October 1922 became assistant of Orso Mario Corbino, director of the Physics Institute of Via Panisperna in Rome. In the same period Corbino met Fermi and was struck by the young physicist, immediately recognizing his potentiality for the renewal of Italian physics. Fermi's early contributions to relativity theories and his competence in the new physics were appreciated by mathematicians like Levi-Civita and Volterra - who were especially committed to the application of mathematics to physical problems - and were fundamental in constructing the high esteem in which they held the young physicist. They also invited him to talk at the Mathematical Seminar of Rome University. In the meantime Persico, too, was building strong relations with the mathematical group. During the academic year 1922-1923 Persico took notes of Levi-Civita's Course on tensor calculus, and edited such lectures, published under the title Lezioni di calcolo differenziale assoluto (an English edition of the first ever treatise on this topic was later promoted by E. T. Whittaker) and also published his own theoretical contributions to GR.

Thanks to the firm support of the mathematicians - notably Levi-Civita, Severi, and Castelnuovo - as well as of Armellini, Corbino succeeded in having a chair of theoretical physics established for the first time in Italy. Both Fermi and Persico won the competition and were appointed respectively in Rome and in Florence in fall 1926, opening the age of modern physics in Italy. 
However, both Fermi and Persico stopped doing research in the field of general and special relativity already in 1923 and directed their attention to atomic physics and quantum theory, topics that were becoming much more pressing for theoretical enquiries. Levi-Civita, too, did not publish anything on GR from 1923 to 1929. This waning of interest in GR was a reflection of a more general international trend, which Jean Eisenstaedt called "the low-water mark" period of GR, in which there was a substantial decline of interest in the theory - a period that went roughly from the mid-1920s to the mid-1950s. In the 1930s, the GR tradition in Rome was kept alive by Levi-Civita, who in 1929 restarted doing important research on the advancement of the theory. The most relevant of these studies, was perhaps his work on the $n$-body problem in GR, which he pursued from 1936 to 1938.

However, dramatic political developments disrupted Levi-Civita's activities. According to the Law Number 1390 of September 5, 1938 (the so-called Legislative measures in defense of the race in the Fascist school), Jews were excluded from the Italian school system as well as from academies and cultural institutions. The effects of the Racial Laws were more than catastrophic for Italian science as a whole. The Italian school of physics was wiped out (this was what Edoardo Amaldi called "the disaster of Italian Physics"). After Fermi left for US in 1938, Amaldi, who had been Fermi's collaborator in the 1930s, struggled to maintain the position gained by the group in the international scene. After the war, Amaldi became a leading figure in the development and reconstruction of Italian and European physics.

\section{The Second Phase: The Renaissance of GR}

The renaissance of GR in Rome in the postwar period is to be understood in two different contexts. The first one is the context of the postwar reconstruction of Italian science, which was driven by some scholars who assured a certain continuity even during the dramatic events related to the war. The second context is the international process related to the revival of interest in the theory of GR. Starting from the 1950s, Einstein's theory of gravitation began to gain momentum for several complex interconnected reasons, including a diffused recognition that the resolution of problems related to GR-proper was essential for different research fields such as quantum gravity, unified field theories, and cosmology. Similarly to what happened elsewhere, the revival of research in GR in Rome was shaped by the confluence of various research agendas pursued in different disciplinary contexts. The first of these elements, in temporal order, was the renewal of interest in GR within the mathematical community, in strong connection with the tradition inaugurated and pursued by Levi-Civita. The main actor of the re-establishment of this tradition was Carlo Cattaneo. A former student of Levi-Civita's in Rome, Cattaneo had been appointed to the chair of Rational Mechanics in Pisa in 1949. Probably influenced by his frequent intellectual exchanges with André Lichnerowicz, Cattaneo began to be strongly interested in the development of mathematical methods in GR around 1957. The international leaders of the field soon recognized in Cattaneo the main 
representative of GR in Italy, as shown by the fact that he became a member of the International Committee on General Relativity and Gravitation as soon as it was established in 1959. The same year, Cattaneo came back to Rome University, where he was appointed to the chair of Rational Mechanics. Since the beginning of the 1960s, Cattaneo actively promoted the study of GR in Italy and, more specifically, in Rome, establishing his school. Starting from 1958 he organized several Summer Schools and Conferences dedicated to GR. Moreover, in parallel with Rational Mechanics, Cattaneo began also teaching a course entitled Relativistic Theories: a complementary exam included in all study areas of the Mathematics courses. At the same time, he also created a research group dedicated to GR, which was made possible by an institutional re-organization of mathematical research in Italy. In 1959 it was decided to establish and fund fifty research groups in Mathematics within the CNR, the National Research Council. When these groups began operating in 1960, the 36th group, headed by Cattaneo, focused on "Einstein's theory of gravitation".

Besides the mathematical school, other elements flew into the renaissance of GR. These other elements were all connected with the re-construction of physics research in Italy after the disruption of WWII - a process driven by a few authoritative scholars, notably Edoardo Amaldi, who took the role of organizers of Italian physics. In this context, and in connection with the decision of unveiling of secret research on thermonuclear fusion at an international level, in 1957 Amaldi and Persico founded the Laboratorio Gas Ionizzati (LGI), dedicated to research on fusion and plasma physics. The LGI shortly became the natural context to bring in dialogue experts of different disciplines and to create the environment for interdisciplinary projects. The co-presence of several actors in the same institutional space significantly contributed to the renewal of interest in research fields related to GR.

At the same time, Amaldi began promoting the organization of space activities in Italy and in Europe. The new perspectives deriving by radioastronomical research during the 1950s, and by the growing importance of what at the end of the decade was being called "cosmic physics" did not escape Amaldi's attention. He realized that Astrophysics was dramatically absent in Rome, and took the decision to call back from Argentina the astrophysicist Livio Gratton, who had emigrated there immediately after the war. Like Cattaneo, Gratton had relevant connections with the interwar research on GR in Rome. (In 1931, he had graduated in Rome under Armellini's supervision with a thesis on relativistic cosmology - a topic that had been proposed by Castelnuovo). In 1960, Amaldi used the funds of LGI to have Gratton in Rome. The following year Amaldi succeeded in having the first chair of Astrophysics established in Rome. The new appointment gave Gratton the institutional support to propose the creation of a Laboratory of Astrophysics connected with his chair at the Physics Institute, already at the beginning of 1962.

Gratton had a central role in sparkling a new kind of research agenda in astrophysics in Italy, which naturally brought to connections with GR. He was one of the first Italian scholars to recognize the great importance of relativistic astrophysics 
and a large part of early research of the group was within this field. His interest was especially focused on very dense objects, like neutron stars - on which he lectured at the first Texas Symposium on Quasars and Relativistic Astrophysics in 1963, a conference that inaugurated the era of relativistic astrophysics and boosted a new interest in GR. Gratton also invited for long periods foreign visitors like Geza Szamosi and young brilliant physicists graduated in other universities, like Alvio Renzini and Giancarlo Setti from Bologna. Excellent students like Franco Pacini, Franco Occhionero, Alvio Renzini, Claudio Firmani, became his collaborators. During the early 1960s, Arrigo Finzi, a former student of Levi-Civita, worked with Gratton making innovative works in astrophysics and teaching a course on questions related to GR at the Physics Institute.

During the 1960s, the discovery of quasars, Cosmic Microwave Background, and pulsars went hand in hand with the new observational possibilities offered by the X-ray astronomy. Amaldi had longstanding contacts with Bruno Rossi, the pioneer of cosmic-ray research in Italy during the 1930s, who was at the time a leading figure of US space activities and the promoter of X-ray astronomy. All this was instrumental in Gratton's decision to direct a part of the research activity to prepare experiments to be flown on rockets (X-ray telescope, UV spectrographs, participation to the great European astronomical satellite with an X-ray experiment). An experiment on cosmic sources of X rays was prepared by Gratton's group and successfully flown on a NASA rocket in 1969. That same year, a conference was organized in Rome on non-solar X- and gamma-ray astronomy as the 37th symposium of the International Astronomical Union, of which at the time Gratton was the vice-president.

Although it is not clear how the dialogue developed in detail, it appears that Gratton's students began having some connections with Cattaneo and his group since the early 1960s. A first clue in this direction is that Vittorio Cantoni, who had graduated with Gratton, was a member of Cattaneo's group in 1962-63. With time, the connections between the two groups became more relevant, although they seemed to grow without well-established institutional frameworks. Actors' recollections suggest that these collaborations were mainly driven by personal relationships and the recognition of common interests.

In this sense, the trajectories of Franco Pacini and Remo Ruffini are cases in point. After having completed his dissertation on neutron stars with Gratton during the academic year 1963-1964, Pacini began collaborating with Silvano Bonazzola, a young mathematician with strong interests in astrophysics, and a member of Cattaneo's group since its establishment. The Bonazzola-Pacini collaboration briefly preceded Pacini's renowned studies on neutron stars, which made him a world expert on astrophysics of highly compact stars after the discovery of pulsars in 1967. After a long period in the US, in 1978 Pacini was called on a chair at Florence University and nominated director of the Arcetri Observatory, which became one of the leading astrophysical institutes in Europe. A second important contact 
between the groups of astrophysics and mathematical GR in Rome, was established by the physics student Remo Ruffini, who decided to do his degree thesis under Cattaneo's supervision. Ruffini's work was actually closely followed by Bonazzola, who was interested in the development of numerical models studying the equilibrium configurations of a system of self-gravitating scalar bosons or spin- $1 / 2$ fermions a work that made use of Ruffini's expertise with calculators. After his graduation in 1966 and a short period with Pascual Jordan in Hamburg, in September 1967 Ruffini moved to Princeton and began collaborating with John A. Wheeler, shortly becoming a leading figure in relativistic astrophysics.

The institutional structures of Cattaneo's group, the LGI and the Laboratory of Astrophysics made possible a series of other interdisciplinary collaborations. Bonazzola continued to work in astrophysics collaborating with Bruno Bertotti and Alfonso Cavaliere, both working at the Laboratory of Ionized Gases. Bertotti was also an internationally recognized expert of GR with a thesis on unified field theory, and a long international experience with Wheeler in Princeton - where he wrote a much cited work (later known as the Bertotti-Robinson solution). Bertotti was called back by Amaldi to work in the LGI and lecture on magnetohydronynamics, which was important both for fusion research and for astrophysical plasmas. Moreover, both the LGI and the Astrophysical laboratory were located in Frascati, providing an occasion for Bertotti and Cavaliere to interact with Pacini, too.

Although mathematics and astrophysics were the two central disciplines involved in the renaissance of GR in Rome, there were other institutional contexts that provided significant elements that fed into the renaissance of GR. The most important, was the group on space research on interplanetary plasmas, directed by Guido Pizzella. His first PhD student was Francesco Melchiorri. With his wife Bianca Olivo he began developing detectors that would be later used for the first experiments on the Cosmic Microwave Background. This relevant research project, that had been suggested by Rossi himself, initiated observational cosmology in Italy.

During the second half of the 1960s, difficulties arose for Gratton's group from the discontinuous and insufficient funding. Some of his collaborators emigrated because of the impossibility to find a proper position in Italy. Many of them, however, fertilized other national and international institutions.

In the meantime, however, astrophysics had acquired a relevant status in Rome. Moreover, a group had formed around the project for the search of gravitational waves led by Pizzella and supported by Amaldi, who pursued this kind of research for the rest of his life. In the late 1970s, the Institute became attractive enough to rebuild a strong group of researchers, especially around the figure of Remo Ruffini. He was appointed the chair of theoretical physics in Rome in 1978, where he introduced the problems of relativistic astrophysics to many generations of students. In 1981 Francesco Melchiorri, too, came back from Florence, bringing with him his group of brilliant students, who have since then brilliantly continued his fundamental work on the Cosmic Microwave Background. 